\title{
Universal Aspects of Deconfinement: Interfaces, Flux Tubes and Self-Duality in 2+1 Dimensions
}

\author{
Lorenz von Smekal* \\ Institut für Kernphysik, Technische Universität Darmstadt, D-64289 Darmstadt, Germany \\ E-mail: lorenz.smekal@physik.tu-darmstadt.de
}

\section{Sam R. Edwards}

Institut für Kernphysik, Technische Universität Darmstadt, D-64289 Darmstadt, Germany

E-mail: edwards@crunch.ikp.physik.tu-darmstadt.de

\section{Nils Strodthoff}

Institut für Kernphysik, Technische Universität Darmstadt, D-64289 Darmstadt, Germany

E-mail: nstrodt@crunch.ikp.physik.tu-darmstadt.de

\begin{abstract}
We study center vortex free energies and 't Hooft's electric fluxes on the lattice in $2+1$ dimensions, where $S U(2)$ for example, is in the universality class of the $2 d$ Ising model. This places a wealth of exact results at our fingertips. In particular, spacelike center vortices in $S U(2)$ near criticality correspond to spin interfaces in the $2 d$ Ising model, whose universal scaling functions are known exactly. We exploit this to locate the deconfinement transition with unprecedented precision and subsequently for a finite size scaling analysis, where the self-duality of the $2 d$ spin model is reflected in a duality between the spacelike vortices and confining electric fluxes. The corresponding relation between the string tension and its dual in the high temperature phase is arguably the simplest example of a universal amplitude ratio. Around the transition, both can be efficiently extracted from the exact results with a global one-parameter fit which allows straightforward continuum extrapolation.
\end{abstract}

The XXVIII International Symposium on Lattice Field Theory, Lattice2010

June 14-19, 2010

Villasimius, Sardinia, Italy

\footnotetext{
* Speaker.
} 


\section{Introduction}

The finite temperature deconfinement transition in pure $S U(N)$ gauge theories in $d+1$ dimensions is very well understood in terms of the spontaneous breakdown of their global $Z_{N}$ center symmetry. This symmetry is faithfully represented by the fundamental Polakov loops which live on $d$ dimensions and develop a non-zero expectation value in the deconfined broken phase much like the spontaneous magnetization in a $d$ dimensional $q$-state Potts model [1] with $q=N$.

In order to be able to apply the powerful tools of universality and scaling near a critical point, here we are particularly interested in cases where this transition is of $2^{\text {nd }}$ order. For pure QCD with $N=3$ colors in $3+1$ dimensions this is of course not the case. The transition is first order, but only just. If we either reduce the number of colors to $N=2$ or the dimensions to $2+1$, we obtain YangMills theories with a second order deconfinement transition within the universality class of $q$-state Potts models with $q=2$ (Ising) in $d=3$ dimensions or with $q=2$ and 3 in $d=2$. The $q=4$ Potts model in 2 dimensions is interesting because it is known [2] to have a $2^{\text {nd }}$ order transition and to fall precisely on the separatrix $q_{c}(d)$ with respect to the order of the transition in the $(q, d)$-plane, where only $1^{\text {st }}$ order transitions occur for $q>q_{c}(d)$, i.e., $q_{c}(2)=4$. The corresponding $S U(4)$ gauge theory in $2+1$ dimensions has recently been studied for example in $[3,4,5]$. The conclusion in [4] is that the transition is weakly $1^{\text {st }}$ order, unlike the $q=4$ Potts case. It nevertheless seems that there is a rather wide range of intermediate length scales where at least approximate Potts scaling can be observed. One might then like to understand, for example, why among the wider class of Ashkin-Teller models it is the standard $q=4$ Potts scaling that is relevant here, and whether this can be derived from an effective Polyakov-loop model.

We will present more results specific for $S U(3)$ and $S U(4)$ in $2+1$ dimensions in [6]. One general property of the $2 d$ Potts models is that they are self-dual for all $q$. We will demonstrate below, how this self-duality is reflected in a duality between the spacelike center vortices and the confining electric fluxes of the gauge theory. Herein we will mainly report on numerical results for $S U(2)$ in $2+1$ dimensions for which many exact results from the $2 d$ Ising model are available.

\section{Center Vortices and Interfaces}

For pure $S U(N)$ gauge theories in a finite Euclidean box, 't Hooft's twisted boundary conditions fix the total $Z_{N}$-valued center flux through each plane of the box, i.e., the total number modulo $N$ of center vortices piercing that plane [7]. At finite temperature $T$, the differences in free energy between these twisted ensembles and the periodic one define the corresponding center-vortex free energies. If they tend to zero in the thermodynamic limit, then these vortices condense which leads to an area law for those Wilson loops that feel their disordering phases in the corresponding plane of the twist. In an $L^{d} \times 1 / T$ box, we have to distinguish between temporal and magnetic twist. The latter is defined in purely spatial planes and corresponds to the $Z_{N}$-valued magnetic flux $\vec{m}$ of static center monopoles. Their free energy tends to zero with $L \rightarrow \infty$ as $\exp \left\{-\sigma_{s}(T) L^{2}\right\}$ at all $T$ [8], corresponding to the area law for spatial Wilson loops with spatial string tension $\sigma_{s}(T)$.

Relevant to the deconfinement transition, however, are only the temporal twists. These are characterized by $d$-dimensional vectors $\vec{k}$ of integers $\bmod N$, i.e., with components $k_{i} \in Z_{N}$ representing the twist in the temporal plane of orientation $(0, i)$, with total center flux $\exp \left(2 \pi i k_{i} / N\right)$ through that plane. See Fig. 1 for an illustration of such a vortex ensemble in $S U(2)$. We denote the partition functions of the various ensembles with temporally twisted boundary conditions by $Z_{k}(\vec{k})$. 

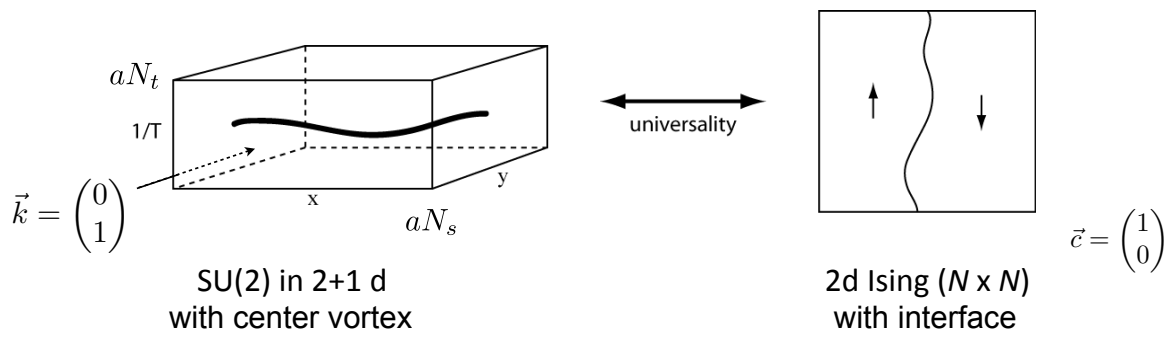

Figure 1: Spatial center vortices share their universal behavior with interfaces in a spin model.

The corresponding center-vortex free energies per $T$ are then given by $F_{k}(\vec{k})=-\ln \left(Z_{k}(\vec{k}) / Z_{k}(0)\right)$, where $Z_{k}(0)$ stands for the periodic ensemble. In the vicinity of a second order deconfinement transition these vortex free energies show the universal behavior of interfaces in the respective $d$ dimensional Potts model. These interface free energies, $F_{I}(\vec{c})=-\ln \left(Z_{q}(\vec{c}) / Z_{q}(0)\right)$, are likewise obtained from ratios of partition functions $Z_{q}(\vec{c})$ with boundary conditions denoted by a $d$ dimensional vector $\vec{c}$ such that the $q$ spin states $s_{\vec{x}}=0,1, \ldots q-1$ are cyclically shifted across the boundary, i.e., $s_{\vec{x}+\vec{e}_{i} L}=s_{\vec{x}}+c_{i} \bmod q$, with $c_{i}=0,1, \ldots q-1$. For $q=2$, the Ising model, this simply means anti-periodic in direction $i$, if $c_{i}=1$, and periodic otherwise.

Interfaces(vortices) are suppressed in the low(high) temperature ordered(deconfinement) phase below(above) $T_{c}$. Here, the ratios $R_{q}(\vec{c}) \equiv Z_{q}(\vec{c}) / Z_{q}(0)$ and $R_{k}(\vec{k}) \equiv Z_{k}(\vec{k}) / Z_{k}(0)$ both tend to zero as we approach the thermodynamic limit. Complementary to that, in the disordered(confined) phase, it is the interface(vortex) free energies that tend to zero such that the ratios $R_{q}, R_{k}$ approach 1 for all boundary conditions. Only at the critical temperature $T=T_{c}$ do these ratios converge to non-trivial and universal fixed points. In 2 dimensions these universal numbers $0<R_{c}^{(m, n)}<1$ have been obtained exactly [9], in terms of Jacobi theta functions, for all cyclic boundary conditions $\vec{c}=(m, n)$ and all Potts models with $2^{\text {nd }}$ order transitions, i.e., for $q=2,3$ and 4 .

These universal ratios can be used to determine the critical couplings $\beta_{c}$ of the transition in the $2+1$ dimensional gauge theories with high precision [10], by requiring that $\beta=\beta_{c}$ at $R_{k}=R_{c}$. For $S U(2)$, where,

$$
R_{c}^{(1,0)}=1 /\left(2^{3 / 4}+1\right), \text { and } R_{c}^{(1,1)}=\left(2^{3 / 4}-1\right) /\left(2^{3 / 4}+1\right),
$$

from the $2 d$ square Ising model, for example, this allowed us to determine the critical couplings for lattices with up to $N_{t}=9$ links in the Euclidean time direction with a typically by two orders of magnitude increased precision where there were literature values available $[11,12,5]$. The high precision allowed us to reliably determine the subleading $1 / N_{t}$-corrections to the linearly increasing behavior of $\beta_{c}$ with $N_{t}$ near the continuum limit [10],

$$
\beta_{c}\left(N_{t}\right)=1.5028(21) N_{t}+0.705(21)-0.718(49) \frac{1}{N_{t}} .
$$

The slope of the leading term determines the critical temperature $T_{c}$ in units of the dimensionful continuum coupling $g_{3}^{2}$ of the $(2+1) d$ theory, yielding $T_{c} / g_{3}^{2}=0.3757(5)$. By standard arguments, from this result one can also read off the temperature dependence of the coupling at a given $N_{t}$,

$$
\beta(t)-\beta_{c}=4 N_{t} \frac{T_{c}}{g_{3}^{2}} t-\frac{0.270(2)}{N_{t}} \frac{g_{3}^{2}}{T_{c}} \frac{t}{1+t}+\mathscr{O}\left(1 / N_{t}^{2}\right), \quad t=\frac{T}{T_{c}}-1 .
$$

For each fixed $N_{t}$, we thus have precise control of the temperature by varying the lattice coupling $\beta$. The physical length of the spatial volume then follows from $L=a N_{s}=T_{c}^{-1}\left(N_{s} / N_{t}\right) /(1+t)$. 
With these we can perform a finite-size-scaling analysis near the $2^{\text {nd }}$ order phase transition where generalized couplings such as the vortex-ensemble ratios $R_{k}$, for sufficiently large $L$, only depend on $L^{1 / v} t$, with the correlation-length critical exponent $v$, in a universal way. In particular, for $(2+1) d S U(2)$, this dependence is determined by that of the corresponding ratios $R_{q=2}$ of finite-volume partition functions of the $2 d$ square Ising model, with $v=1$, which have been obtained exactly for all combinations of periodic and anti-periodic boundary conditions in [13].

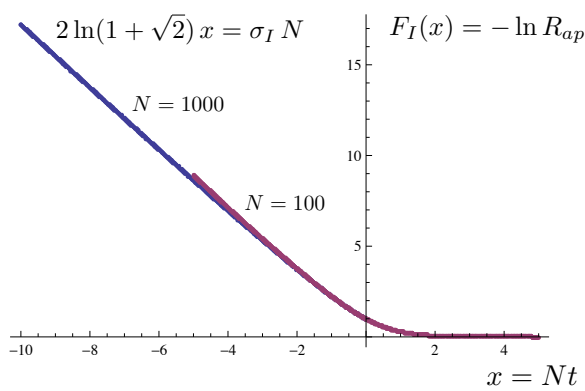

Figure 2: Universal scaling function $F_{I}(x)$ from the exact finite-volume partition functions of the $N \times N$ square Ising model in [13].

For one antiperiodic direction, the corresponding exact interface free energies per temperature $F_{I}=-\ln R_{a p}$, where $R_{a p}$ stands for $R_{q=2}(\vec{c})$ with either $\vec{c}=(1,0)$ or $(0,1)$ which are the same on an $N \times N$ square lattice, are plotted over the finite-size-scaling variable $x \equiv N t$ for $N=100$ and $N=1000$ in Fig. 2. The asymptotic slope in the ordered phase $(t<0)$ for large $N$ approaches $2 \ln (1+\sqrt{2})$ which follows from Onsager's famous result [14] for the tension of a straight interface in the infinite volume limit, $\sigma_{I}=2 K+\ln \tanh K$ with spin-coupling per temperature $K=J / T$, near criticality at $K_{c}=\ln (1+\sqrt{2}) / 2$. Deviations from that, e.g., observed for $N=100$ around $x=-5$, are due to subleading (non-universal) finite-volume effects.

We have calculated the $S U(2)$ vortex-ensemble ratios $R_{k}(\vec{k})$ with the algorithm of [15] as previously for the $(3+1) d$ theory in [16], here in $(2+1) d$ for $N_{t}=2$ to 10 , each with spatial sizes up to $N_{s}=96$ and in a suitable window of $\beta$ 's around $\beta_{c}$ to test the finite-size scaling (FSS). For each fixed $N_{t}$ we generally observe very good scaling of the available data for all $N_{s}$ when plotted over the FSS variable $x \equiv L T_{c} t=\left(N_{s} / N_{t}\right) t(\beta) /(1+t(\beta))$ (assuming $v=1$ for the $2 d$ Ising universality class) with $t(\beta)$ from Eq. (2.3). For the resulting vortex free energies $F_{k}$ (for $\vec{k}=(1,0)$ and $(1,1)$ ) we then obtain accurate one-parameter fits via $F_{k}(x)=F_{I}(-\lambda x)$ to the exact universal scaling functions $F_{I}(x)$ (for $(1,0)$ and $(1,1)$ b.c.'s). This determines the single non-universal parameter $\lambda$ which is dimensionless and relates the $S U(2)$ FSS variable to the Ising one (whereby the minus sign reflects the interchange of high and low temperature phases between the two).

\section{Self-Duality in $2+1$ Dimensions}

The $2 d q$-state Potts models are self-dual for all $q$. This has long been known for infinite systems. A particularly simple proof based on the random bond-cluster representation was given in [1]. Far less is known in a finite volume with translationally invariant boundary conditions, however, where ensembles with different boundary conditions mix under duality transformations. One case where this is known is the $3 d$ Ising/ $Z_{2}$-gauge theory system [17]. For the Potts model, using the random cluster methods developed in [9], we were able to obtain the following exact duality transformations for all $q$ on a finite $2 d$ torus as discrete $2 d$ Fourier transforms over all ensembles $Z_{q}^{(m, n)}$ with cyclically shifted boundary conditions $\vec{c}=(m, n)$,

$$
Z_{q}^{(-s, r)}(\widetilde{K})=\left(\frac{e^{\widetilde{K}}-1}{e^{K}-1}\right)^{N_{\text {sites }}} \frac{1}{q} \sum_{m, n} e^{\frac{2 \pi i}{q}(r m+s n)} Z_{q}^{(m, n)}(K), \quad m, n, r, s=0,1, \ldots q-1,
$$




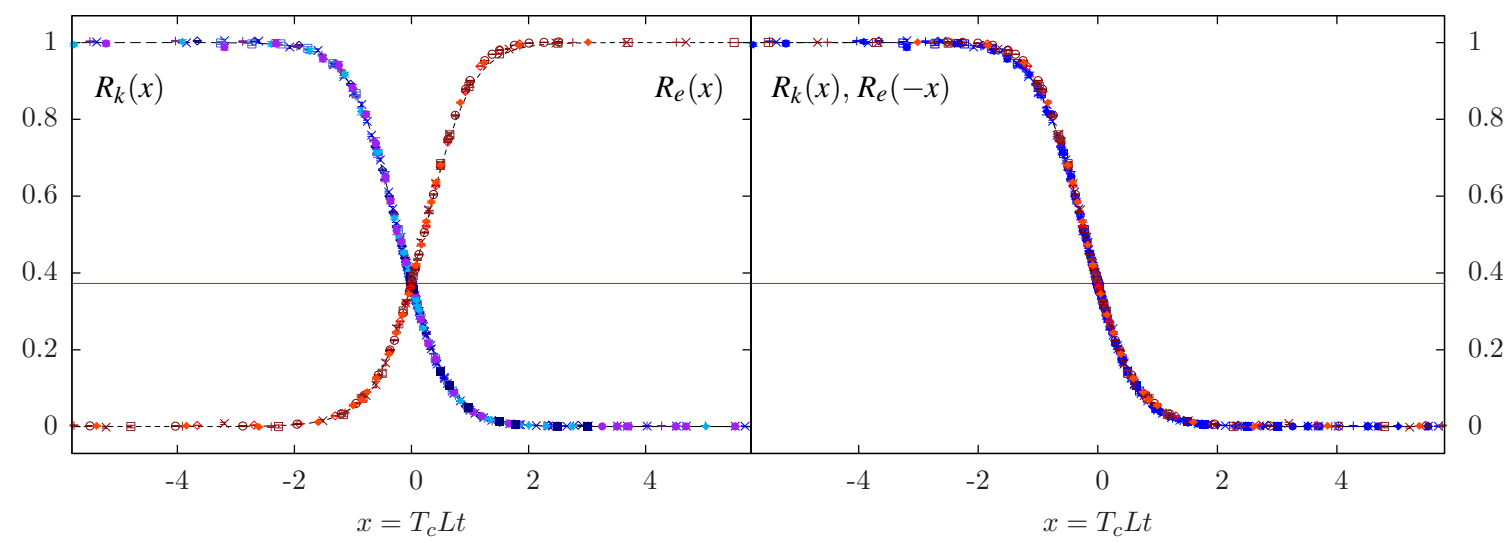

Figure 3: The ratio $R_{k}$ of the partition function with twist $\vec{k}=(1,0)$ over the periodic ensemble compared to that of one unit of electric flux relative to the no-flux ensemble (left), $R_{e}=Z_{e}(\vec{e}) / Z_{e}(0)$ with $\vec{e}=(1,0)$, and to its mirror image (right). The data here is obtained for $N_{t}=4$ and various spatial volumes up to $N_{s}=96$.

where $N_{\text {sites }}$ is the total number of sites of the graph on the $2 d$ torus (i.e., $N^{2}$ on an $N \times N$ square lattice), $K=J / T$ the coupling per temperature, and $\widetilde{K}$ its dual obtained from $\left(e^{\widetilde{K}}-1\right)\left(e^{K}-1\right)=q$, with temperature mirrored around criticality at $K=\widetilde{K}=K_{c}=\ln (1+\sqrt{q})$.

For $q=2$, and with $K \rightarrow K / 2$, conventionally, this relation reduces to an analogous known result for the Ising model [18]. For $q=3$ it agrees with a corresponding result [19] obtained for the planar or vector Potts model which is equivalent to the standard one in that case. The proof of the general formula in Eq. (3.1), for all $q$-state Potts models on a $2 d$ torus, will be given elsewhere [6].

Here we only note that the pattern in the finite-volume duality transforms, by the discrete Fourier transform over the ensembles with cyclically shifted boundary conditions, is precisely the same as that between the twisted vortex ensembles $Z_{k}(\vec{k}, \vec{m})$ in the $d+1$ dimensional pure $S U(N)$ gauge theory and those with fixed units of electric (and magnetic) flux $Z_{e}(\vec{e}, \vec{m})$ [7],

$$
Z_{e}(\vec{e}, \vec{m})=\frac{1}{N^{d}} \sum_{\vec{k} \in Z_{N}^{d}} e^{2 \pi i \vec{e} \cdot \vec{k} / N} Z_{k}(\vec{k}, \vec{m}),
$$

obtained from a $d$-dimensional $Z_{N}$-Fourier transform over all temporally twisted $\vec{k}$-b.c.'s. We are not interested in magnetic flux and drop the argument $\vec{m}$ again, in the following. The role of the electric flux ensembles is best understood in terms of the translationally invariant flux between a fundamental color charge at some point $\vec{x}$ in the finite volume and its mirror charge at $\vec{x}+\vec{e} L$ in a neighboring volume in the direction of the flux $\vec{e}$ [16], it contains no ultraviolet divergent perimeter and no short-distance Coulomb contributions [20],

$$
Z_{e}(\vec{e}) / Z_{e}(0)=\left\langle P(\vec{x}) P^{\dagger}(\vec{x}+\vec{e} L)\right\rangle_{\text {no-flux }},
$$

where the $P$ 's are untraced fundamental Polyakov loops, including any non-trivial transition functions in the time direction, and the subscript indicates that their expectation value is taken in the enlarged no-flux ensemble $Z_{e}(0)=\sum_{\vec{k}} Z_{k}(\vec{k}) / N^{d}$. Without loss, the form in (3.3) assumes periodic spatial b.c.'s and is manifestly invariant under spatially periodic gauge transformations.

Of course, the duality relations provide exact maps between the high and low temperature phases in the spin models, valid at all temperatures and beyond universality. We do not have such exact duality relations for the gauge theories, but near criticality, where $K \leftrightarrow \widetilde{K}$ amounts to $x \leftrightarrow-x$, we expect that the spin model dualities are reflected in analogously dual relations between 

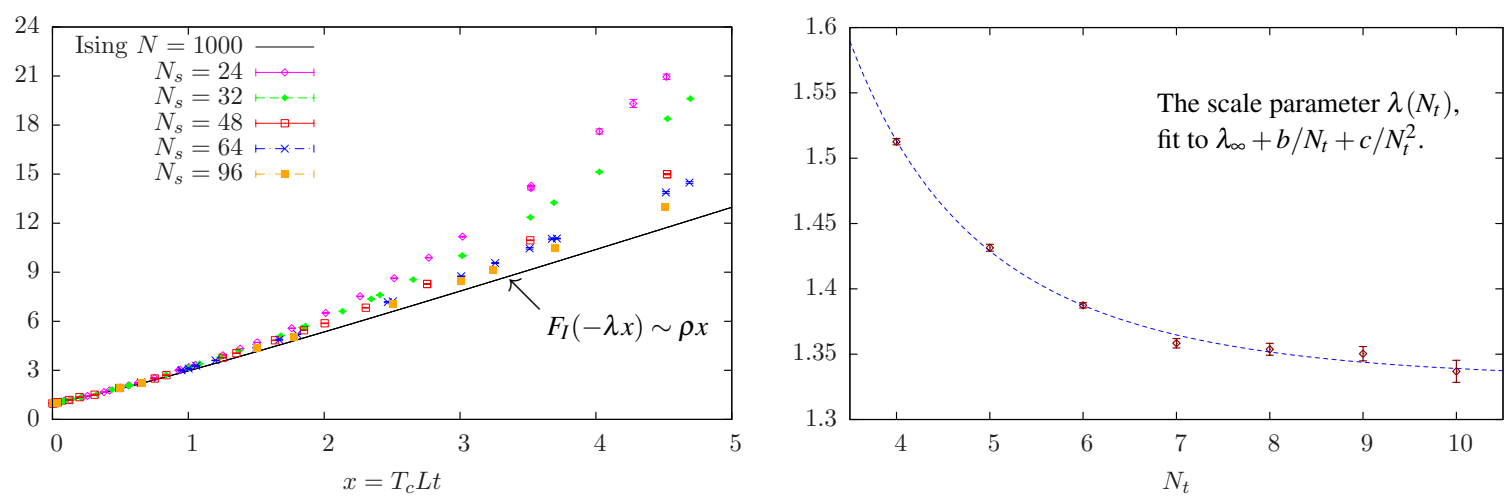

Figure 4: Vortex free energy $F_{k}(x)$ in $S U(2)$ above $T_{c}$ (left), and $\lambda\left(N_{t}\right)$ from fits to $F_{k}(x)=F_{I}(-\lambda x)$ (right).

the behavior of the spatial center vortices and the confining electric fluxes. This is indeed the case in a rather wide universal scaling window around $T_{c}$ and, in particular, for $2+1 d$ gauge theories the self-duality of the spin models implies that the electric-flux free energies $F_{e}(\vec{e})$ are mirror images of the center vortex free energies, i.e., $F_{e}(x)=F_{k}(-x)$, as demonstrated for $S U(2)$ in Fig. 3.

\section{Universal Amplitude Ratios and Continuum Limit}

The interface tension $\sigma_{I}$ in the ordered phase of the spin model corresponds to the dual string tension $\tilde{\sigma}$ for spatial center-vortex sheets which provides the dual area law for the spatial 't Hooft loops above $T_{c}$. Both vanish near the $2^{\text {nd }}$ order phase transition, e.g., $\tilde{\sigma} \sim t^{\mu}$ for $t \rightarrow 0^{+}$, with a critical exponent that is related to the correlation length critical exponent $v$ via the hyperscaling relations as $\mu=(d-1) v$, i.e. $\mu=v$ in $d=2$ spatial dimensions. The product of interface tension and correlation length (to the power $d-1$ ) is an example of a universal amplitude ratio [21]. If we use the exponential correlation length in the disordered phase of the spin model, $\xi_{\text {gap }}^{+}$, then the universal constant given by $\sigma_{I}\left(\xi_{\text {gap }}^{+}\right)^{d-1}=R_{\sigma \text { gap }}^{+}$turns out to be exactly 1 for $q=2,3$ and probably also for $q=4$ in 2 dimensions. In the gauge theory the exponential correlation length of Polyakov loops in the confined phase is given by string tension and temperature as $(\sigma / T)^{-1}$. Therefore, we have a universal relation between the string tension below and the dual string tension above $T_{c}$,

$$
\tilde{\sigma}=R_{\sigma \text { gap }}^{+}(\sigma / T)^{d-1}, \text { or } \sigma=\rho T_{c}^{2}(-t)^{v}+\cdots \text { and } \tilde{\sigma}=R_{\sigma \text { gap }}^{+}\left(\rho T_{c}\right)^{d-1} t^{(d-1) v}+\cdots,
$$

where at leading order in the reduced temperature $t$ the only unknown is the single unique constant $\rho$ that we have introduced here. This constant can be extracted from the vortex free energies in the high temperature phase at asymptotically large $x=T_{c} L t^{v}$, where $F_{k}(x)=R_{\sigma \text { gap }}^{+} \rho^{d-1} x^{d-1}$, by direct numerical simulations. This was done in [16]. It is numerically very hard and expensive because larger and larger lattices are needed to stay within the universal scaling window as $x$ is increased.

This is demonstrated for $(2+1) d S U(2)$ in Fig. 4 where the universal scaling function is known exactly from the Ising model. While its linear large- $x$ behavior, $F_{k}(x) \rightarrow \rho x$, is approached with increasing lattice size, even for the $N_{s}=96$ lattice it is still difficult to extract the asymptotic slope reliably from the data. But we know the asymptotic slope of the universal scaling function $F_{I}(x)$ which is $2 \ln (1+\sqrt{2})$. Therefore, from $F_{k}(x)=F_{I}(-\lambda x)$ we obtain $\rho=2 \lambda \ln (1+\sqrt{2})$ with $\lambda$ from our one-parameter fits at small $x$ where we have very accurate data. Moreover, we have determined this single non-universal parameter for $N_{t}=4,5 \ldots 10$ which allows a polynomial fit, $\lambda\left(N_{t}\right)=\lambda_{\infty}+b / N_{t}+c / N_{t}^{2}$, with the extrapolated result $\lambda_{\infty}=1.354(25)$. This then determines the 
leading behavior of the continuum string tension and its dual around the phase transition,

$$
\sigma=\lambda T_{c}^{2} 2 \ln (1+\sqrt{2})|t|+\cdots, t \rightarrow 0^{-}, \text {and } \tilde{\sigma}=\lambda T_{c} 2 \ln (1+\sqrt{2}) t+\cdots, t \rightarrow 0^{+} .
$$

\section{Conclusions}

Here we presented results for center vortex free energies in the $2+1$ dimensional $S U(2)$ gauge theory whose universal properties near the deconfinement transition can be analyzed in terms of many exact results from the $2 d$ Ising model. We used these to precisely locate the transition, to demonstrate finite-size-scaling, and to extract the continuum string tension and its dual from universal amplitude ratios together with one-parameter fits to exactly known universal scaling functions.

We found an exact duality transformation for the $q$-state Potts models on a finite $2 d$ torus and demonstrated that their self-duality is reflected in the $(2+1) d$ gauge theory: The free energies of spatial center vortices are mirror images around $T_{c}$ of those of the confining electric fluxes.

More results for $S U(3)$ and $S U(4)$ will be presented elsewhere, see also [6]. The relevance of center symmetry and center vortices for confinement in full QCD when including the electromagnetic interactions of the fractionally charged quarks are discussed in [22].

Acknowledgements: This work was supported by the Helmholtz International Center for FAIR within the LOEWE program of the State of Hesse, the Helmholtz Association Grant VH-NG-332, and the European Commission, FP7-PEOPLE-2009-RG No. 249203. Simulations were performed on the high-performance computing facilities of eResearch SA, South Australia.

\section{References}

[1] F. Y. Wu, Rev. Mod. Phys. 54 (1982) 235 [Erratum-ibid. 55 (1983) 315].

[2] R. J. Baxter, J. Phys. C C 6 (1973) L445.

[3] Ph. de Forcrand and O. Jahn, Nucl. Phys. Proc. Suppl. 129 (2004) 709.

[4] K. Holland, M. Pepe and U. J. Wiese, JHEP 0802 (2008) 041.

[5] J. Liddle and M. Teper, arXiv:0803.2128.

[6] N. Strodthoff, S. Edwards and L. von Smekal, PoS Lattice2010 (2010) 288;

N. Strodthoff, S. Edwards and L. von Smekal, in preparation.

[7] G. 't Hooft, Nucl. Phys. B 153 (1979) 141.

[8] L. von Smekal and Ph. de Forcrand, Nucl. Phys. Proc. Suppl. 119 (2003) 655.

[9] H. Park and M. den Nijs, Phys. Rev. B 38 (1988) 565.

[10] S. Edwards and L. von Smekal, Phys. Lett. B 681 (2009) 484.

[11] M. Teper, Phys. Lett. B 313 (1993) 417.

[12] J. Engels, et al., Nucl. Phys. Proc. Suppl. 53 (1997) 420.

[13] M.-C. Wu, M.-C. Huang, Y.-P. Luo and T.-M. Liaw, J. Phys. A: Math. Gen. 32 (1999) 4897.

[14] L. Onsager, Phys. Rev. 65 (1944) 117.

[15] Ph. de Forcrand, M. D'Elia and M. Pepe, Phys. Rev. Lett. 86 (2001) 1438.

[16] Ph. de Forcrand and L. von Smekal, Phys. Rev. D 66 (2002) 011504(R).

[17] M. Caselle, M. Hasenbusch, P. Provero and K. Zarembo, Nucl. Phys. B 623 (2002) 474.

[18] G. Münster, private communication (2001); A. I. Bugrij and V. N. Shadura, arXiv:hep-th/9601106.

[19] A. I. Bugrii and V. N. Shadura, arXiv:hep-th/9706045.

[20] Ph. de Forcrand and L. Von Smekal, Nucl. Phys. Proc. Suppl. 106 (2002) 619.

[21] A. Pelissetto and E. Vicari, Phys. Rept. 368 (2002) 549.

[22] S. Edwards, A. Sternbeck and L. von Smekal, PoS Lattice2010 (2010) 275. 